\title{
Pyloric reflux and the healing of gastric ulcers
}

\author{
J. B. COCKING AND P. GRECH \\ From the Northern General Hospital, Sheffield
}

SUMmaRY Pyloric reflux is rare in healthy subjects but is common in those with benign gastric ulcer. Healing of a gastric ulcer is associated with a diminution of reflux, and occasionally the pylorus becomes fully competent.

There is considerable evidence that reflux of duodenal contents into the stomach is rare in healthy people whereas it is common in those with benign gastric ulcer (Du Plessis, 1965; Capper, Airth, and Kilby, 1966; Rhodes, Barnado, Phillips, Rovelstad, and Hofmann, 1969; Flint and Grech, 1970; Wormsley, 1972). The significance of this observation is not clear, but it has been suggested that reflux is injurious to the gastric mucosa, producing gastritis and subsequent ulceration (Delaney, Cheng, Butler, and Ritchie, 1970). In favour of this theory, Black, Roberts, and Rhodes (1971) found that no consistent changes occurred in the intragastric concentrations of bile acids after gastric ulcers had healed.

In this paper we report the effect of the healing of gastric ulcer on pyloric reflux, using a radioopaque medium.

\section{Material and Methods}

The technique used has already been described by Grech (1970). Twenty $\mathrm{ml}$ of a 1 in 3 micropaque suspension was injected into the duodenal cap through a fine catheter with the patient supine. Five minutes later the amount of barium that had refluxed into the stomach was graded in the following manner: mild reflux $(+)$, no fluid level visible in the stomach in the erect position and only a small amount of mucosal coating in the antrum when supine; moderate reflux $(++)$, a fluid level produced when erect, and partial coating of the gastric mucosa when supine; marked reflux $(+++)$, the gastric mucosa completely coated in the supine position.

When the first injection of barium had cleared from the stomach and duodenum a second $20 \mathrm{ml}$ was injected into the duodenal cap with the patient

Request for reprints to J. B. Cocking, Isle of Thanet District Hospital, Ramsgate Wing, Ramsgate, Kent.

Received for publication 3 May 1973. standing, and five minutes later the amount refluxing into the stomach was graded using the same criteria. The whole procedure, including the grading of reflux, was carried out under fluoroscopy with an image intensifier. The final amount of reflux was recorded by conventional radiography.

Ten patients with benign gastric ulcer, diagnosed by barium meal and confirmed endoscopically, were studied. In each case the ulcer was situated in the middle third of the lesser curve. None had additional organic disease of the pylorus or duodenum, nor did they have coexisting chronic chest disease (Beeley and Grech, 1971). They were compared with 10 normal controls matched for age and sex. There were four males and six females in the ulcer and control groups. The average age of each group was 53 years $( \pm 2.9$ SEM). Four patients in the ulcer group and two in the control group smoked more than 10 cigarettes per day. Each patient with a gastric ulcer had an initial pyloric reflux test performed, and was then treated with carbenoxolone sodium $50 \mathrm{mg}$ tds and antacids as an outpatient. Smoking was discouraged. When the ulcer had healed as observed, both radiographically and endoscopically, a second reflux test was performed. All reflux tests were carried out on patients who had fasted overnight.

Three other patients with benign mid lesser curve gastric ulcers and additional organic pyloroduodenal disease were also studied in a similar manner. Patients $A$ and $B$ had chronic duodenal ulcers and patient $\mathbf{C}$ had a radiographical deformity of the antrum due to scarring, as confirmed by endoscopy and multiple biopsies.

\section{Results (see Table)}

None of the control group had pyloric reflux, in either the erect or the supine position, whereas all the patients with gastric ulcer had marked or moderate reflux in the erect position before treatment. After 555 


\begin{tabular}{|c|c|c|c|c|c|c|c|}
\hline \multirow[t]{3}{*}{ Patient } & \multirow[t]{3}{*}{$\operatorname{Sex}$} & \multirow[t]{3}{*}{ Age } & \multirow{3}{*}{$\begin{array}{l}\text { Initial Ulcer } \\
\text { Diameter }(\mathrm{cm})\end{array}$} & \multicolumn{4}{|l|}{ Reflux } \\
\hline & & & & \multicolumn{2}{|c|}{ Before Treatment } & \multicolumn{2}{|c|}{ After Treatment } \\
\hline & & & & Erect & Supine & Erect & Supine \\
\hline 1 & $\mathbf{F}$ & 44 & 1.0 & +++ & 0 & 0 & 0 \\
\hline 2 & $\mathbf{F}$ & 53 & $0 \cdot 3$ & ++ & 0 & + & 0 \\
\hline 3 & $\mathbf{F}$ & 54 & 0.5 & ++ & 0 & + & 0 \\
\hline 4 & $\mathbf{F}$ & 54 & 1.5 & ++ & 0 & + & 0 \\
\hline 5 & $\mathbf{F}$ & 65 & 1.2 & ++ & + & + & 0 \\
\hline 6 & $\mathbf{F}$ & 68 & $0 \cdot 8$ & ++ & 0 & + & 0 \\
\hline 7 & $\mathbf{M}$ & 41 & 1.0 & +++ & 0 & 0 & 0 \\
\hline 8 & $\mathbf{M}$ & 42 & 0.8 & ++ & 0 & + & 0 \\
\hline 9 & $\mathbf{M}$ & 53 & 1.0 & ++ & 0 & + & 0 \\
\hline 10 & $\mathbf{M}$ & 59 & 1.0 & ++ & 0 & + & 0 \\
\hline \multicolumn{8}{|c|}{ With organic pyloroduodenal disease } \\
\hline A & $\mathbf{F}$ & 55 & $1 \cdot 2$ & +++ & + & ++ & + \\
\hline B & $\mathbf{M}$ & 34 & 0.5 & ++ & + & + & 0 \\
\hline C & $\mathbf{M}$ & 67 & $1 \cdot 0$ & +++ & + & +++ & 0 \\
\hline
\end{tabular}

Table Effect of gastric ulcer healing on pyloric reflux

healing of the ulcers, eight of the ulcer group had mild reflux when erect, and in two the pylorus had become competent. Changes in reflux did not correlate with initial ulcer size.

Patients A and B both showed diminution of reflux after their ulcers had healed but in neither did the pylorus become competent. Patient $\mathrm{C}$ with pyloric scarring had marked reflux in the erect position before and after healing, though the mild reflux seen in the supine position before healing was not present after treatment.

\section{Discussion}

These observations show that healing of a gastric ulcer is associated with a diminution of duodenogastric reflux. The radiographic technique that was used gives a crude measurement of the volume of dilute barium that refluxes in a given time; however, it is not known if the latter has any quantitative relationship with the actual volumes of duodenal contents that reflux. The method is also open to the criticisms that intubation of the pylorus may affect reflux and inaccuracies in the quantitation of reflux may arise from observer error. However, in this study any inaccuracies attributable to methodology applied in the main to controls and ulcer patients alike, although it was impossible to exclude unconscious bias from the observations, as they could not be made in a blind manner. Nevertheless we believe that the effect of observer error on the results was small, as the three defined categories of reflux differentiated marked changes in pyloric incompetence. Any intermediate degree of reflux was allocated to the lower of the two alternative categories, so that the decrease in reflux that occurred with the healing of the ulcers tended to be underestimated.

The most obvious explanation for the results of this study is that the duodeno-gastric reflux arose secondarily to the gastric ulceration. In support of this hypothesis Garrett, Summerskill, and Code (1966) reported that the antral motility decreased in the presence of a gastric ulcer and returned to normal after healing had taken place. They explained these findings by postulating that the ulcer produced an interruption between action potentials and the contractile mechanism of antral smooth muscle. One assumes that the failure of the pylorus to become competent when the gastric ulcers healed was related to residual scar tissue in the ulcer site. However, this particular explanation of our observations is contrary to much current thought, and ignores the increasing evidence that suggests that duodeno-gastric reflux produces mucosal damage, gastritis, and ultimately ulceration (Delaney, Cheng, Butler, and Richie, 1970).

The latter concept does not explain what initiates reflux and why it diminishes as gastric ulcers heal. One could postulate that the causative factors are removed by treatment or that treatment itself reduces the degree of pyloric incompetence. However, little is known about the pathogenesis of reflux. It may be aggravated by smoking (Rhodes, 1972), it is affected by posture, being greater in the erect than in the supine position, and is frequently found in association with organic disease of the pyloro-duodenal region (Flint and Grech, 1970). Forty per cent of the ulcer group in this series smoked, and smoking was discouraged during treatment; thus smoking may have been partially responsible for the development of reflux at the outset, and reduction in smoking may 
have resulted in diminution in reflux in a proportion of the patients. Changes in posture were unlikely to explain the observed decrease in reflux, as the patients were treated ambulantly when reflux is greatest. Nor was organic disease of the juxta-pyloric region a factor producing reflux in the 10 patients comprising the ulcer group, as it was carefully excluded by barium examination and endoscopy. In addition there is to date no evidence that carbenoxolone sodium has any direct action on the pylorus to reduce reflux. This hypothesis does not therefore give a satisfactory explanation for our findings.

If duodeno-gastric reflux is an important aetiological factor in the pathogenesis of gastric ulceration, our results could best be explained by postulating that the reflux arose initially as a result of severalas yet unidentified-factors, and that the resulting gastric ulcer affected gastric motility to produce a temporary increase in reflux. Rhodes (1972) has reviewed how bile will break the gastric mucosal barrier and allow hydrogen ions to penetrate and damage the mucosa. However, there is little information as to whether this damage is specifically related to the volumes or the concentrations of the refluxing duodenal contents or whether it is dependent on the relative proportions of the gastric, biliary, and pancreatic secretions present in the stomach. The concentrations of the intragastric bile acids vary inversely with the volumes of pancreatic and gastric secretions with which they mix, so that it is quite possible to have a reduction in the volume of duodeno-gastric reflux with no alteration in the intragastric concentrations of the bile acids. This explains the apparent discrepancy between the results of this study and those of Black et al (1971). They assessed changes in reflux by measuring intragastric bile acid concentrations and concluded that ulcer 'healing did not affect bile reflux in any consistent way'; in contrast the present study was entirely concerned with volume changes in reflux. The fact that intragastric bile acid concentrations remain constant whilst the volume of reflux diminishes when a gastric ulcer heals points to concentration rather than volume of intragastric bile being the more important factor determining whether or not gastric ulceration is likely to develop. This is supported by the additional observation of Black and his colleagues (1971) that intragastric bile acid concentrations are generally higher in subjects with gastric ulcers than in normal controls. However, further information is required before more definite conclusions can be formulated about this aspect.

We wish to thank Dr F. J. Flint for his help and advice, and the other physicians of the Northern General Hospital, Sheffield, who allowed us to investigate the patients under their care.

\section{References}

Beeley, M., and Grech, P. (1971). Pyloric incompetence in chronic non-specific lung disease. Gut, 12 102-106.

Black, R. B., Roberts, G., and Rhodes, J. (1971). The effect of healing on bile reflux in gastric ulcer. Gut, 12, 552-558.

Capper, W. M., Airth, G. R., and Kilby, J. O. (1966). A test for pyloric regurgitation. Lancet, 2, 621-623.

Delaney, J. P., Cheng, J. W. B., Butler, B. A., and Ritchie, W. P., Jr. (1970). Gastric ulcer and regurgitation gastritis. Gut, 11, 715-719.

Du Plessis, D. J. (1965). Pathogenesis of gastric ulceration. Lancet, 1, 974-978.

Flint, F. J., and Grech, P. (1970). Pyloric regurgitation and gastric ulcer. Gut, 11, 735-737.

Garrett, J. M., Summerskill, W. H. J., and Code, C. F. (1966). Antral motility in patients with gastric ulcer. Amer. J. dig. Dis., 11, 780-789.

Grech, P. (1970). A technique for assessing pyloric reflux. Gut, 11, 794-795.

Rhodes, J. (1972). Etiology of gastric ulcer. Gastroenterology, 63, 171-182.

Rhodes, J., Barnado, D. E., Phillips, S. F., Rovelstad, R. A., and Hofmann, A. F. (1969). Increased reflux of bile into the stomach of patients with gastric ulcer. Gastroenterology, 57, 241-252.

Wormsley, K. G. (1972). Aspects of duodeno-gastric reflux in man. Gut, 13, 243-250. 